\title{
Chronic oral corticosteroids use and persistent eosinophilia in severe asthmatics from the Belgian severe asthma registry
}

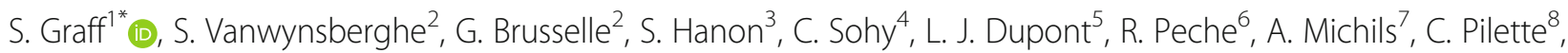
G. Joos ${ }^{2}$, R. E. Louis ${ }^{1}$ and F. N. Schleich ${ }^{1}$

\begin{abstract}
Background: Severe asthma (SA) may require frequent courses or chronic use of oral corticosteroids (OCS), inducing many known side effects and complications. Therefore, it is important to identify risk factors of chronic use of OCS in SA, considering the heterogeneity of clinical and inflammatory asthma phenotypes. Another aim of the present analysis is to characterize a subpopulation of severe asthmatics, in whom blood eosinophil counts (BEC) remain elevated despite chronic OCS treatment.

Methods: In a cross-sectional analysis of 982 SA patients enrolled in the Belgian Severe Asthma Registry (BSAR) between March 2009 and February 2019, we investigated the characteristics of the OCS treated patients with special attention to their inflammatory profile.

Results: At enrollment, 211 (21\%) SA patients were taking maintenance OCS (median dose: 8 [IQR: 5-10]) mg prednisone equivalent). BEC was high $\left(>400 / \mathrm{mm}^{3}\right)$ in $44 \%$ of the OCS treated population. Multivariable logistic regression analysis showed that risk factors for chronic use of OCS in SA were late-onset asthma (i.e. age of onset $>$ $40 \mathrm{yr}$ ), frequent exacerbations (i.e. $\geq 2$ exacerbations in the previous year) and non-atopic asthma. Late-onset asthma was also a predictor for persistently high BEC in OCS treated SA patients.

Conclusion: These data showed a significant association between a persistently high BEC and late-onset asthma in OCS treated SA patients. Whether it is poor compliance to treatment or corticosteroid insensitivity the reasons for this association warrants further investigation.
\end{abstract}

Keywords: Asthma, Blood eosinophils, Oral corticosteroids

Although severe asthma (SA) represents only $5-10 \%$ of the population with asthma [1], the condition accounts for a major part of the financial burden to health care systems posed by asthma [2]. More than half of the

\section{* Correspondence: sgraff@uliege.be}

'Department of Respiratory Medicine, CHU Sart-Tilman, I3GIGA Research Group, University of Liege, Liege, Belgium

Full list of author information is available at the end of the article incremental cost has been recently attributed to comorbidities [3].

SA defined according to a joint statement from the European Respiratory Society and the American Thoracic Society (ERS/ATS) may require frequent or chronic use of oral corticosteroids (OCS). Indeed, OCS are prescribed as maintenance therapy in $45 \%$ of adult SA patients who remain uncontrolled despite GINA step 4-5 treatment [4]. 
However, OCS are responsible for serious side effects [5] and complications accounting for a significant economic burden $[6,7]$.

OCS effectively target elements of the T2 inflammatory pathway, suppressing blood eosinophils quite rapidly [8] and decreasing mast cells in airway smooth muscle [9]. Yet some patients still present with high blood eosinophil levels $\left(\geq 400 / \mathrm{mm}^{3}\right)$ despite OCS maintenance therapy.

The goals of the present analysis were to compare patients with and without OCS maintenance, and to characterize patients with high levels of blood eosinophils despite this treatment. For this purpose, we used the Belgian Severe Asthma Registry (BSAR), which is a cohort of adult severe asthmatics and aims at enhancing awareness and knowledge on the natural history of SA in order to optimize patient care [10].

\section{Methods}

In a cross-sectional analysis on SA patients enrolled in the BSAR, we compared characteristics of SA patients chronically using OCS with those SA patients who did not. Chronic OCS use was defined as daily use of OCS. Furthermore, we made a comparison of patients with high $\left(\geq 400 / \mathrm{mm}^{3}\right)$ and low blood eosinophil counts (BEC) under OCS.

We also looked at a small number of severe asthmatics with persistently elevated BEC despite OCS therapy in whom an anti-IL5 monoclonal antibody was started.

Between March 2009 and February 2019, severe asthmatics [1] from 21 Belgian centers were enrolled in the BSAR, utilizing a secured web database which admits password protected anonymized data [10], after gaining fully informed written consent (2008/221). The American Thoracic Society definition of SA [11] was used for patients recruited between 2009 and 2014. As the definition of SA changed in 2014 [1], the BSAR website was adapted accordingly.

Asthma was diagnosed based on symptoms of cough, breathlessness or dyspnea together with the demonstration of airflow variability [12]. The latter was defined by one or more of the following: increase in forced expiratory volume in $1 \mathrm{~s}$ (FEV1) of $12 \%$ or greater after inhalation of $400 \mu \mathrm{g}$ of salbutamol or an inhaled concentration of methacholine provoking a $20 \%$ fall in FEV1 of less than $16 \mathrm{mg} / \mathrm{ml}$. Methacholine challenges were performed according to a standardized methodology as previously described [13].

Other prerequisites for inclusion were: age $\geq 18$ years, asthma follow-up by a respiratory physician for at least 12 months, education on the disease provided to the patient, and compliance thought to be satisfactory in order to include only patients with severe refractory asthma and those in whom comorbidities had been addressed. All the data presented were collected at the time point of recruitment into the registry.

Patients were characterized as atopic if they had at least one positive specific IgE (>0.35 kU/l; ImmunoCAP system, Phadia AB, Uppsala; Sweden) to at least one common aeroallergen (cat, dog, house dust mites, grass pollen, tree pollen and a mixture of molds) or a positive skin prick test. An exacerbation in the previous year was defined by a course of OCS for at least 3 days for asthma worsening. Emergency room visits, hospitalizations, and intensive care unit stays were also recorded. Presence (or absence) of treatment with long-acting muscarinic antagonists (LAMA), short or long-acting 32 -agonists (SABA, LABA), anti-IgE, anti-IL5 and macrolides was recorded, as well as OCS maintenance doses.

Quality of life was assessed using the self-administered Asthma Quality of Life Questionnaire (AQLQ) [14] and asthma control by both the Juniper Asthma Control Questionnaire (ACQ) [15] and the Asthma Control Test (ACT) [16].

Patients underwent FENO measurement at a flow rate of $50 \mathrm{ml} / \mathrm{s}$ according to the ERS/ATS recommendations (NIOX, Aerocrine, Sweden) [17] followed by spirometry with bronchodilation (inhalation of $400 \mu \mathrm{g}$ salbutamol) and complete pulmonary function tests with evaluation of lung volumes and diffusion capacity.

Tobacco status were recorded. An ex-smoker was defined as someone who had stopped smoking for at least 6 months.

Sputum was induced almost exclusively in Liège and processed as previously reported [18]; 225 sputum samples were collected in the data set $(23 \%$ of the total population). Cell counts were estimated on samples centrifuged (Cytospin) and stained with Hemacolor ${ }^{\circ}$ Staining set after counting 500 non-squamous cells (Merck chemical, Overijste, Belgium). Sputum cytology was analyzed and 4 phenotypes were defined: the eosinophilic phenotype with $\geq 3 \%$ sputum eosinophil count (and $<$ $76 \%$ neutrophil count), the neutrophilic phenotype with $\geq 76 \%$ sputum neutrophil count (and $<3 \%$ eosinophil count), and the mixed granulocytic phenotype being a combination of the above [19]. The fourth was paucigranulocytic phenotype, defined as an inflammatory cell count below these thresholds. Blood samples were drawn for evaluation of total serum IgE levels, specific IgE and BEC.

Nasal polyps and sinusitis were diagnosed by Ear Nose and Throat specialists either by endoscopy or sinus CT scanner. Gastroesophageal reflux was diagnosed either upon anamnesis (symptoms of heartburn), or by the presence of oesophagitis upon gastroscopy or treatment response to PPI. Chest CT imaging was obtained in patients with an atypical presentation of SA and in case of 
history of smoking. Hospital Anxiety and Depression Scale (HADS) questionnaires [20] were used to assess psychopathology.

Data on vocal cord dysfunction, allergic bronchopulmonary aspergillosis, Aspirin-sensitivity, eosinophilic granulomatosis with polyangiitis (EGPA: formerly called Churg-Strauss syndrome), occupational asthma (asthma acquired in the workplace), premenstrual asthma, and obesity were also collected.

Near-fatal asthma is described as an asthma attack that require intensive care unit stay with mechanical ventilation.

BEC were categorized in 4 groups: $<150 / \mathrm{mm}^{3}$, between 150 and $299 / \mathrm{mm}^{3}$, between 300 and $400 / \mathrm{mm}^{3}$, and $>400 /$ $\mathrm{mm}^{3}$ to evaluate eosinophil levels in patients treated with OCS and the mean dose of OCS in each group.

Finally, an analysis was conducted to compare patients normalizing their BEC $\left(<400 / \mathrm{mm}^{3}\right)$ under OCS treatment versus those who did not.

\section{Statistical methods}

Continuous variables are presented as mean and SD when normally distributed or as median and interquartile range (IQR) when not normally distributed. Categorical variables were presented as frequencies and percentages. Continuous variables were compared by ttest when normally distributed or by Wilcoxon MannWhitney test when non-parametric. ANOVA or Kruskal Wallis analyses were conducted in order to compare more than 2 groups (categories). Fisher exact test was used to compare qualitative variables.

Predicting factors of "chronic OCS use" were assessed by a logistic regression using independent variables such as atopy, gender, age, body mass index, age of asthma onset, smoking status, pack-year, and measurements of ACQ, FENO, post-bronchodilation $\mathrm{FEV}_{1} \%$, sputum \% eosinophils and neutrophils, BEC, presence of exacerbations, and hospitalizations during the last year and comorbidities such as nasal polyps, rhinosinusitis, bronchiectasis, emphysema, obesity, GERD, allergic bronchopulmonary aspergillosis, vocal cord dysfunction, aspirin-sensitivity, EGPA, psychopathy, occupational, premenstrual, or Aspergillar asthma. Chronic OCS use was used as the dependent variable. After examining for all potential predictors, the univariate association with the outcome, a stepwise backward logistic regression was conducted; initial model included variables with an association of $p<0.20$. Then, the best predictive model was conducted deleting those variables that had the weakest association with the outcome $(p>0.05)$. This analysis (logistic regression) was repeated in order to identify predictors of $B E C \geq 400 \mathrm{~mm}^{3}$.

A $p$ value $<0.05$ was considered statistically significant. Statistical analysis was done using STATA version 14.0 (Statistical Software, College Station, TX: StataCorp LP).

\section{Results}

Between March 2009 and February 2019, 982 severe asthmatics that fulfilled the definition of SA $[1,11]$ were enrolled in the BSAR.

\section{Characteristics of OCS maintenance treated SA patients}

Demographic, functional and inflammatory characteristics and comorbidities of SA patients are shown in Table 1. At enrollment in the BSAR, 211 (21\%) SA patients were taking maintenance OCS (median dose (IQR): 8 (5-10) $\mathrm{mg}$ prednisone equivalent/day). The proportion of males was higher $(p=0.02)$ in SA patients treated with maintenance OCS and these patients significantly more often had late-onset $(\geq 40 \mathrm{yr})$ asthma $(p<0.0001)$, were less atopic $(p<0.0001)$, and presented with higher FENO $(p=0.03)$ and higher exacerbation rates $(p<0.0001)$ compared to OCS naïve SA patients. OCS-treated SA patients also had worse asthma control $(p=0.03)$ and asthma-related quality of life scores $(p=$ $0.03)$. Moreover, comorbidities such as emphysema ( $p=$ $0.002)$, bronchiectasis $(p=0.001)$, GERD $(p=0.007)$ and EGPA $(p<0.0001)$ were more prevalent in the chronic OCS-treated SA group.

Interestingly, BEC were similar $(p=0.2)$ between OCS-treated and not treated SA patients with $44 \%$ patients with high BEC $(>400 / \mathrm{mm} 3)$ in the OCS-treated group (Fig. 1). Even in SA patients treated with the highest dose of OCS (>16 mg/d of prednisone equivalent), $27 \%$ still had high BEC.

When selecting only patients on biologics (anti-IL5 or anti-IgE) $(n=270)$ (Table S1), the proportion of males was higher $(p=0.001)$ in SA patients treated with maintenance OCS and these patients were less atopic $(p=0.005)$, and presented with higher exacerbation rates $(p=0.0009)$ compared to OCS naïve SA patients. OCS-treated SA patients also had worse asthma control $(p=0.03)$. Moreover, sputum eosinophils were lower $(p=0.02)$ and sputum neutrophils higher $(p=0.005)$ in the OCS treated patients.

\section{Predictors of OCS maintenance use}

The results of the univariate logistic regression analysis showed an increased risk of using maintenance OCS in non-atopic male and between 44- and 64-years old SA patients with late-onset asthma (i.e. age of onset $\geq 40 \mathrm{yr}$ ), frequent exacerbations (i.e. $\geq 2$ exacerbations in last 12 months), GERD and EGPA.

Multivariable logistic regression analysis (Table 2) showed that late-onset asthma (OR [95\%CI]:1.98 [1.273.08], $p=0.003$ ), frequent exacerbations (OR [95\%CI]: 3.73 [2.41-5.77], $p<0.0001)$ and non-atopic status $(0.59$ [0.42-0.84], $p=0.003$ ) were predictive of chronic use of OCS in SA. 
Table 1 Comparison of SA patients with or without chronic OCS treatment $(n=982)$

\begin{tabular}{|c|c|c|c|}
\hline Characteristics & No chronic OCS use & Chronic OCS use & $P$-value \\
\hline N (\%) & $771(79)$ & $211(21)$ & $\mathrm{N} / \mathrm{A}$ \\
\hline Gender: $\mathrm{F}(\%)(n=982)$ & $463(60)$ & $107(51)$ & 0.018 \\
\hline Age $(n=981)$ & $53 \pm 16$ & $55 \pm 17$ & 0.0792 \\
\hline \multicolumn{4}{|l|}{ Age of onset (\%) $(n=982)$} \\
\hline$<12 \mathrm{yr}$ & $241(32)$ & $41(20)$ & \multirow[t]{3}{*}{$<0.0001$} \\
\hline $12-40 y r$ & $296(39)$ & $71(34)$ & \\
\hline$\geq 40 \mathrm{yr}$ & $222(29)$ & $97(46)$ & \\
\hline BMI $(n=982)$ & $28 \pm 13$ & $27 \pm 5$ & 0.2454 \\
\hline \multicolumn{4}{|l|}{ Smoking History: $(n=982)$} \\
\hline Never (\%) & $471(61)$ & $126(60)$ & \multirow[t]{3}{*}{0.092} \\
\hline Current (\%) & $62(8)$ & $9(4)$ & \\
\hline Ex (\%) & $238(31)$ & $76(36)$ & \\
\hline Estimated number of packyears $(n=386)$ & $15(7-25)$ & $15(7-25)$ & 0.7406 \\
\hline Atopic status: y (\%) $(n=982)$ & $554(72)$ & $119(56)$ & $<0.0001$ \\
\hline Respiratory Familial History of asthma: true $(\%)(n=982)$ & $348(45)$ & $70(33)$ & 0.001 \\
\hline \multicolumn{4}{|l|}{ Current housing: $(n=975)$} \\
\hline City (\%) & $241(31)$ & $57(28)$ & \multirow[t]{3}{*}{0.201} \\
\hline Countryside (\%) & $281(37)$ & $70(34)$ & \\
\hline Sub-urban (\%) & $246(32)$ & $80(39)$ & \\
\hline SABA: y $(\%)(n=982)$ & $656(85)$ & $176(83)$ & 0.3080 \\
\hline LABA: y $(\%)(n=982)$ & $755(98)$ & $211(100)$ & 0.4520 \\
\hline LAMA: y (\%) $(n=982)$ & $20(3)$ & $8(4)$ & 0.2380 \\
\hline \multicolumn{4}{|l|}{ OCS dose (median IQR) (\%) } \\
\hline$<4$ & N/A & $29(14)$ & \multirow[t]{4}{*}{ N/A } \\
\hline $4-8$ & & $76(36)$ & \\
\hline $8-16$ & & $72(34)$ & \\
\hline$>16 \mathrm{mg} / \mathrm{d}$ & & $34(16)$ & \\
\hline Anti-IL5: y $(\%)(n=982)$ & $63(8)$ & $36(17)$ & $<0.0001$ \\
\hline Anti-lgE: y (\%) $(n=982)$ & $140(18)$ & $31(15)$ & 0.1410 \\
\hline Macrolides: y (\%) $(n=982)$ & $4(1)$ & $1(1)$ & 0.7070 \\
\hline $\mathrm{ACT}(n=758)$ & $14.0 \pm 5.3$ & $12.9 \pm 5.2$ & 0.0195 \\
\hline ACQ $(n=642)$ & $2.5 \pm 1.3$ & $2.8 \pm 1.4$ & 0.0319 \\
\hline AQLQ $(n=686)$ & $4.2 \pm 1.4$ & $3.9 \pm 1.4$ & 0.0259 \\
\hline Exacerbations in last 12 months $(n=966)$ & $2(0-3)$ & $3(1-4)$ & $<0.0001$ \\
\hline$\geq 3$ OCS burst for asthma exacerbation in last 12 months $(n=966)$ & $235(31)$ & $128(60)$ & $<0.0001$ \\
\hline Number of emergency visits in last year $(n=340)$ & $1(0-1)$ & $1(0-2)$ & 0.1177 \\
\hline Number of hospitalizations in last year $(n=337)$ & $1(0-1)$ & $1(0-1)$ & 0.0690 \\
\hline Near fatal episodes last year $(n=99)$ & $0(0-0)$ & $0(0-1)$ & 0.2762 \\
\hline Death: $(\%)(n=982)$ & $5(0.6)$ & $5(2.4)$ & 0.043 \\
\hline $\mathrm{FEV}_{1}(\mathrm{~L})(n=953)$ & $2.01 \pm 0.81$ & $1.97 \pm 0.86$ & 0.5144 \\
\hline $\mathrm{FEV}_{1}(\%$ predicted $)(\mathrm{n}=953)$ & $69 \pm 21$ & $67 \pm 23$ & 0.1874 \\
\hline FVC $(\%$ predicted $)(n=953)$ & $87 \pm 19$ & $87 \pm 23$ & 0.7476 \\
\hline $\mathrm{FEV}_{1} / \mathrm{FVC}(\%$ predicted $)(\mathrm{n}=953)$ & $64 \pm 12$ & $62 \pm 13$ & 0.0508 \\
\hline FEV ${ }_{1}$ Reversibility ( $n=793$ ) & $11 \pm 14$ & $12 \pm 13$ & 0.1299 \\
\hline
\end{tabular}


Table 1 Comparison of SA patients with or without chronic OCS treatment $(n=982)$ (Continued)

\begin{tabular}{|c|c|c|c|}
\hline Characteristics & No chronic OCS use & Chronic OCS use & $P$-value \\
\hline Total Lung Capacity $(n=838)$ & $103(91-115)$ & $102(89-113)$ & 0.3674 \\
\hline Functional Residual Capacity $(n=692)$ & $115(96-141)$ & $117(98-138)$ & 0.9700 \\
\hline Residual Volume $(n=782)$ & $137(110-167)$ & $136(107-171)$ & 0.4914 \\
\hline DLCO $(\%)(n=700)$ & $83 \pm 21$ & $80 \pm 19$ & 0.1038 \\
\hline KCO (\%) $(n=672)$ & $98 \pm 21$ & $97 \pm 20$ & 0.6176 \\
\hline PC20M Value $(\mathrm{mg} / \mathrm{ml})(n=88)$ & $1.1(0.26-8.00)$ & $0.34(0.10-2.00)$ & 0.1338 \\
\hline Total serum lgE (kU/l) $(n=818)$ & $182(66-506)$ & $162(66-366)$ & 0.3237 \\
\hline Blood Eosinophils $\left(/ \mathrm{mm}^{3}\right)(n=800)$ & $290(120-526)$ & $339(110-685)$ & 0.2006 \\
\hline \multicolumn{4}{|l|}{ Blood Eosinophils $\left(/ \mathrm{mm}^{3}\right)(n=800)$} \\
\hline$<150$ & $192(30)$ & $52(31)$ & \multirow[t]{4}{*}{0.071} \\
\hline $150-300$ & $133(21)$ & $22(13)$ & \\
\hline $300-400$ & $81(13)$ & $21(12)$ & \\
\hline$>400$ & $225(36)$ & $74(44)$ & \\
\hline Sputum eosinophils (\%) $(n=225)$ & $7.2(1.4-37.0)$ & $6.0(0.4-21.0)$ & 0.1753 \\
\hline Sputum neutrophils (\%) $(n=225)$ & $51(29-73)$ & $63(35-80)$ & 0.3453 \\
\hline \multicolumn{4}{|l|}{$\begin{array}{l}\text { Sputum inflammatory }(n=225) \\
\text { Phenotypes: }\end{array}$} \\
\hline Paucigranulo & $18 \%$ & $19 \%$ & \multirow[t]{4}{*}{0.450} \\
\hline Eosino ( $\geq 3 \%)$ & $60 \%$ & $49 \%$ & \\
\hline Neutro $(\geq 76 \%)$ & $17 \%$ & $26 \%$ & \\
\hline Mixed & $5 \%$ & $7 \%$ & \\
\hline Exhaled NO (50 ml/sec) (ppb) $(n=689)$ & $24(13-47)$ & $30(17-58)$ & 0.0269 \\
\hline Emphysema $(n=971)$ & $65(9)$ & $23(11)$ & 0.002 \\
\hline Bronchiectasis $(n=971)$ & $109(14)$ & $42(20)$ & 0.001 \\
\hline Rhinosinusitis $(n=971)$ & $396(52)$ & $118(56)$ & 0.463 \\
\hline Nasal polyposis $(n=971)$ & $193(26)$ & $66(31)$ & 0.178 \\
\hline Overweight or obesity $(n=971)$ & $393(52)$ & $100(48)$ & 0.507 \\
\hline Psychopathology $(n=971)$ & $140(18)$ & $39(19)$ & 0.630 \\
\hline GERD $(\underline{n}=971)$ & $262(34)$ & $94(45)$ & 0.007 \\
\hline Vocal Cord Dysfunctions ( $n=292$ ) & $1(0.5)$ & $1(1)$ & 0.371 \\
\hline $\operatorname{ABPA}(n=971)$ & $31(4)$ & $11(5)$ & 0.679 \\
\hline Aspirine-sensitive asthma $(n=971)$ & $46(6)$ & $18(9)$ & 0.323 \\
\hline EGPA (Churg Strauss) $(n=971)$ & $13(2)$ & $14(7)$ & $<0.0001$ \\
\hline Occupational asthma $(n=971)$ & $24(3)$ & $7(3)$ & 0.1950 \\
\hline Premenstrual asthma $(n=971)$ & $6(1)$ & $1(0.5)$ & 0.936 \\
\hline Aspergillar asthma $(n=292)$ & $7(3)$ & $4(6)$ & 0.312 \\
\hline
\end{tabular}

Data are presented as mean \pm SD or median and IQR. BMI Body Mass Index, SABA short acting beta-2 agonists, LABA long acting beta-2 agonists, LAMA long acting muscarinic antagonists, OCS oral corticosteroids, FEV 1 forced expiratory volume in $1, F V C$ forced vital capacity, TLC total lung capacity, FRC functional residual capacity, $R V$ residual volume, $D L C O$ diffusing capacity of lung for carbon monoxide, $K C O$ transfer coefficient of the lung for carbon monoxide, $P C 20 M$ provocative concentration of metacholine causing a $20 \%$ fall in FEV 1 ppb, parts per billion, NO nitric oxide, ppb, parts per billion, ACT Asthma Control Test, ACQ Asthma Control Questionnaire, AQLQ Asthma Quality of Life Questionnaire, GERD gastroesophageal reflux disease, ABPA allergic bronchopulmonary aspergillosis, EGPA Eosinophilic granulomatosis with polyangiitis, N/A not applicable

Characteristics of eosinophilic $\left(B E C \geq 400 / \mathrm{mm}^{3}\right) \mathrm{SA}$ patients treated with maintenance OCS

In the population of OCS treated patients, a comparison was made between patients with $\mathrm{BEC} \geq 400$ and $<400$ $\mathrm{mm}^{3}$.
Demographic, functional and inflammatory characteristics and comorbidities of the 79 OCS-treated SA patients with high $\mathrm{BEC}\left(\geq 400 / \mathrm{mm}^{3}\right)$ were compared to those with low BEC in Table 3. Despite similar OCS doses, eosinophilic patients significantly more often had 


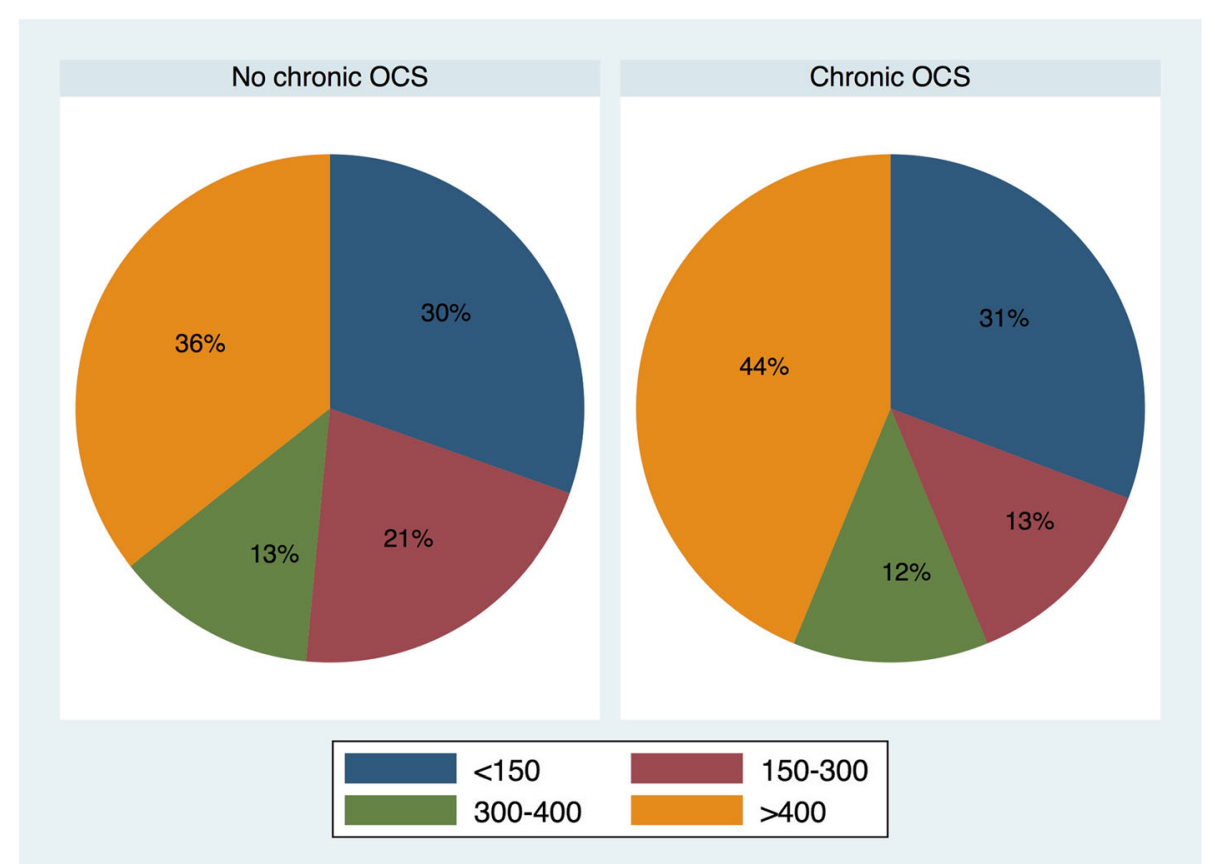

Fig. 1 Blood eosinophil count in SA: Proportions of BEC in severe asthmatics according to (presence or absence of) chronic OCS treatment. BEC categories: $<150 ; \geq 150-<300 ; \geq 300-<400 ; \geq 400\left(/ \mathrm{mm}^{3}\right)$

late-onset asthma $(p<0.0001)$ (Fig. 2), higher levels of FENO $(p=0.0005)$, as well as higher eosinophil $(p=$ $0.02)$ and lower neutrophil $(p=0.0004)$ counts in their sputum than the SA group with a low BEC. These eosinophilic patients also had a higher prevalence of nasal polyposis $(p=0.04)$ and a lower prevalence of anxiety and depression $(p=0.004)$. Moreover, patients with low $\mathrm{BEC}$ had slightly poorer lung function (lower $\mathrm{FEV}_{1}(p=$ $0.04)$ and $\mathrm{FVC} \%(p=0.009)$ but similar $\mathrm{FEV}_{1} / \mathrm{FVC}$ and also poorer asthma control $(\mathrm{p}=0.04)$ than the group with a high BEC. These patients also had a higher prevalence of emphysema $(p=0.02)$.

\section{Predictors of high BEC $\left(\geq 400 / \mathrm{mm}^{3}\right)$ in OCS treated SA patients}

The results of the univariate logistic regression analysis showed an increased risk of being eosinophilic despite maintenance OCS in patients with late-onset asthma, high $\mathrm{FEV}_{1} \%$, high FENO (>50 ppb), and increased sputum eosinophils $(>21 \%)$, but decreased risk are seen in patients with higher sputum neutrophils. Multivariable logistic regression analysis (Table 4) showed that lateonset asthma (OR (IC95\%): 31.6 (3.6-276.9), $p=0.0047$ ) was a predictor for persistently high BEC in OCS-treated SA patients. However, a high ACQ score (>3.9) was inversely associated with high $\mathrm{BEC}$ (OR (IC95\%): 0.17 (0.05-0.63), $p=0.0458)$. Indeed, compared to patients with ACQ scores lower than 3.9 (75th percentile), patients with an ACQ score higher than $3.9(n=31)$ were characterized by low BEC, impaired lung function (low $\mathrm{FEV}_{1}$ ) and diffusing capacity of lung for carbon monoxide (DLCO), and a higher rate of emphysema (52\%) suggestive of Asthma COPD overlap.

\section{BEC before and after anti-IL5 treatment initiation}

In a sub-analysis, 12 patients treated with OCS and persistently elevated BEC $\left(\geq 400 / \mathrm{mm}^{3}\right)$ (594 (535-821)/ $\mathrm{mm}^{3}$ ), were started on an anti-IL5 monoclonal antibody after enrolment in BSAR. We observed normalization $\left(\mathrm{BEC}<400 / \mathrm{mm}^{3}\right)$ of BEC $\left(62(39-91) / \mathrm{mm}^{3}\right)$ in all these patients after one year of anti-IL5 therapy. Also, as previously shown, the OCS dose was significantly reduced from $8(7-10)$ to $4(2-7) \mathrm{mg}$ daily $(p=0.0065)$ after addition of the biologic therapy.

\section{Discussion}

In this study, we found that approximately one-fifth of SA patients included in BSAR was treated with maintenance OCS and that a high proportion (44\%) of these patients still showed a high BEC. The data indicated that late-onset asthma, frequent exacerbations and nonatopic status are associated with OCS maintenance use in SA. Late-onset asthma was also a predictor for persistently high BEC despite OCS.

At enrollment in the registry, 21\% of SA patients were taking maintenance OCS, which is a lower proportion of severe asthmatic than previously described by Chung 
Table 2 Factors associated with maintenance OCS use. Results of the logistic regression - Multivariable analysis - Backward stepwise predictive model $(p<0.2)$

\begin{tabular}{|c|c|c|c|c|c|c|}
\hline \multirow[t]{3}{*}{ Chronic OCS use } & \multicolumn{6}{|c|}{ Total population $(n=953)$} \\
\hline & \multicolumn{3}{|c|}{ UNIVARIATE } & \multicolumn{3}{|c|}{ MULTIVARIATE } \\
\hline & $\overline{O R}$ & $95 \% \mathrm{Cl}$ & $P$-value & $\overline{\mathrm{OR}}$ & $95 \% \mathrm{Cl}$ & $P$-value \\
\hline Atopy & 0.51 & $0.37-0.69$ & $<0.0001$ & 0.59 & $0.42-0.84$ & 0.0030 \\
\hline \multicolumn{7}{|l|}{ Age of onset } \\
\hline$(<12)$ & & & $<0.0001$ & & & 0.0034 \\
\hline$\geq 12 ;<40$ & 1.41 & $0.93-2.15$ & & 1.20 & $0.77-1.86$ & \\
\hline$\geq 40$ & 2.57 & $1.71-3.86$ & & 1.98 & $1.27-3.08$ & \\
\hline \multicolumn{7}{|l|}{ Exacerbations in last 12 months } \\
\hline$(<0)$ & & & $<0.0001$ & & & $<0.0001$ \\
\hline$\geq 0 ;<2$ & 1.16 & $0.73-1.87$ & & 1.16 & $0.72-1.89$ & \\
\hline$\geq 2$ & 3.73 & $2.44-5.70$ & & 3.73 & $2.41-5.77$ & \\
\hline Female & 0.68 & $0.50-0.93$ & 0.015 & & & NS \\
\hline \multicolumn{7}{|l|}{ Smoking (Never) } \\
\hline Current & 0.54 & $0.26-1.12$ & 0.1020 & & & NS \\
\hline Ex & 1.19 & $0.86-1.65$ & & & & \\
\hline \multicolumn{7}{|l|}{ Age $(<44)$} \\
\hline$\geq 44 ;<55$ & 1.70 & $1.09-2.65$ & 0.0385 & & & NS \\
\hline$\geq 55 ;<64$ & 1.86 & $1.18-2.91$ & & & & \\
\hline$\geq 64$ & 1.41 & $0.89-2.23$ & & & & \\
\hline \multicolumn{7}{|c|}{ Number of hospitalizations in last year $(<0)$} \\
\hline$\geq 0 ;<1$ & 1.10 & $0.65-1.85$ & 0.0976 & & & NS \\
\hline$\geq 1$ & 2.01 & $1.04-3.87$ & & & & \\
\hline \multicolumn{7}{|l|}{$\mathrm{FEV}_{1} \%(<53)$} \\
\hline$\geq 53 ;<68$ & 0.70 & $0.46-1.07$ & 0.1845 & & & NS \\
\hline$\geq 68 ;<83$ & 0.64 & $0.41-0.96$ & & & & \\
\hline$\geq 83$ & 0.75 & $0.49-1.16$ & & & & \\
\hline Exhaled NO (50 ml/sec) (ppb) & 1.0049 & $1.00053-1.00948$ & 0.028 & & & NS \\
\hline \multicolumn{7}{|l|}{ Blood eosinophils $\left(/ \mathrm{mm}^{3}\right)(<150)$} \\
\hline$\geq 150 ;<300$ & 0.61 & $0.35-1.05$ & 0.0815 & & & NS \\
\hline$\geq 300 ;<400$ & 0.95 & $0.54-1.69$ & & & & \\
\hline$\geq 400$ & 1.21 & $0.81-1.81$ & & & & \\
\hline Nasal polyps & 1.37 & $0.98-1.93$ & 0.067 & & & NS \\
\hline GERD & 1.59 & $1.16-2.17$ & 0.004 & & & NS \\
\hline ABPA & 1.29 & $0.64-2.62$ & 0.477 & & & NS \\
\hline Aspirin sensitive & 1.47 & $0.83-2.60$ & 0.183 & & & NS \\
\hline EGPA (Churg-Strauss) & 4.21 & $1.94-9.11$ & 0.000 & & & NS \\
\hline
\end{tabular}

$O R$ odds ratio, $C l$ confidence interval, $F E V_{1}$ forced expiratory volume in $1 \mathrm{~s}, N O$ nitric oxide, $p p b$ parts per billion, GERD gastroesophageal reflux disease, $A B P A$ allergic bronchopulmonary aspergillosis, EGPA Eosinophilic granulomatosis with polyangiitis, NS not significant

[1]. A partial explanation for this discrepancy could be that in about $26 \%$ of this subpopulation, a biotherapy (either IL-5 inhibitors or anti-IgE) had already been started prior to enrolment in BSAR. Indeed, OCSsparing effects have been reported to be close to $50 \%$ with these biotherapies [21-23].
Our data confirms the predominance of females in the overall SA population [10]. However, we found a higher proportion of males in severe asthmatics using maintenance OCS when compared to non-OCS treated patients. A higher proportion of males was previously reported in eosinophilic asthma $[24,25]$ and eosinophilic asthma has 
Table 3 Comparison of OCS maintenance SA patients with BEC $<400$ or BEC $\geq 400(n=174)$

\begin{tabular}{|c|c|c|c|}
\hline Characteristics & $\mathrm{BEC}<400$ & $\mathrm{BEC} \geq 400$ & $P$-value \\
\hline N (\%) & $95(55)$ & $79(45)$ & N/A \\
\hline \multicolumn{4}{|l|}{ Age of onset (\%) (yr) $(n=173)$} \\
\hline$<12$ & $25(27)$ & $6(8)$ & \multirow[t]{3}{*}{0.000} \\
\hline $12-40$ & $37(39)$ & $26(33)$ & \\
\hline$>40$ & $32(34)$ & $47(59)$ & \\
\hline ACQ $(n=61)$ & $2.97 \pm 1.29$ & $2.40 \pm 1.37$ & 0.0367 \\
\hline $\mathrm{FEV}_{1}(\%$ predicted $)(n=110)$ & $63.7 \pm 23.6$ & $71.7 \pm 22.4$ & 0.0267 \\
\hline FVC (\% predicted) $(n=110)$ & $83 \pm 21$ & $93 \pm 23$ & 0.0088 \\
\hline Sputum eosinophils (\%) $(n=38)$ & $2.8(0.4-10.0)$ & $46.5(4.7-66.8)$ & 0.0223 \\
\hline Sputum neutrophils (\%) $(n=38)$ & $70.0(54.0-80.0)$ & $21.7(9.5-40.5)$ & 0.0004 \\
\hline Exhaled NO (50 ml/sec) (ppb) $(n=67)$ & $23(13-42)$ & $38(19-83)$ & 0.0005 \\
\hline Emphysema (based on Chest CT Scanner) $(n=174)$ & $15(16)$ & $7(9)$ & 0.025 \\
\hline Nasal polyposis $(n=174)$ & $25(26)$ & $33(42)$ & 0.042 \\
\hline Psychopathology $(n=174)$ & $25(26)$ & $9(11)$ & 0.004 \\
\hline
\end{tabular}

Data are presented as mean \pm SD or median and IQR. FEV forced expiratory volume in $1 \mathrm{~s}, F V C$ forced vital capacity, ppb parts per billion, NO nitric oxide, $p p b$ parts per billion, ACQ Asthma Control Questionnaire, N/A not applicable

been shown to respond well to chronic inhaled corticosteroids and OCS treatment [26-28].

There are different phenotypes of SA. Among these phenotypes, late-onset non allergic asthma has been found to occur after the age of 40 , to be frequently associated with nasal polyps and chronic rhinosinusitis, and to be prone to exacerbations with good response to OCS [25]. In our study, not surprisingly, chronic use of OCS was predicted by late-onset of asthma ( $\geq 40 \mathrm{yr})$, high $(\geq 2)$ exacerbation rates in the previous year, and non-atopic status. Late-onset asthma was also a predictor for high BEC $\left(>400 / \mathrm{mm}^{3}\right)$ in OCS maintenance treated severe

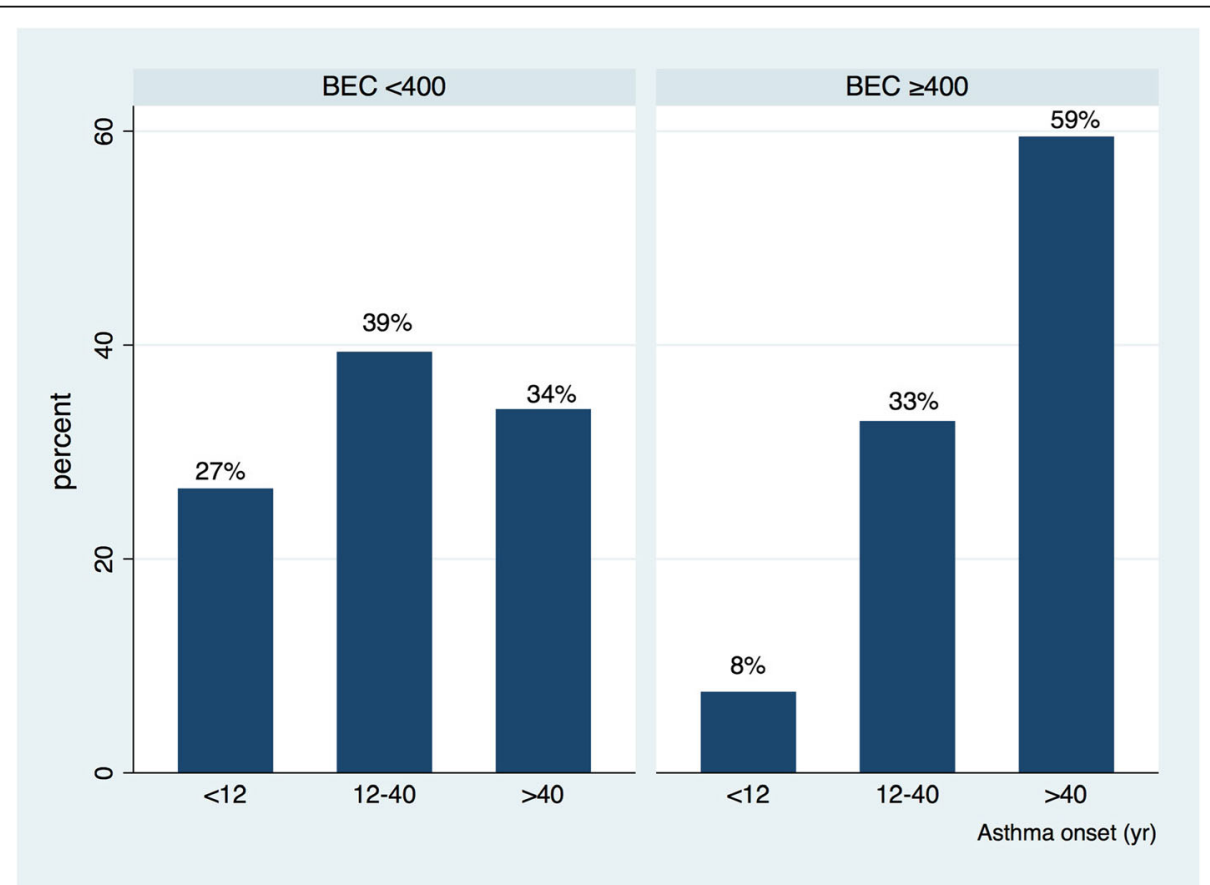

Fig. 2 Late-onset asthma predominance and eosinophilia: Distribution of age onset in severe asthmatic with chronic OCS use according to BEC. Age of asthma onset categories: $<12 ; \geq 12-<40 ; \geq 40$ (yr) 
Table 4 Factors associated with BEC $\geq 400$. Results of the logistic regression - Multivariable analysis - Backward stepwise predictive model $(p<0.2)$

\begin{tabular}{|c|c|c|c|c|c|c|}
\hline \multirow[t]{3}{*}{$\mathrm{BEC} \geq 400$} & \multicolumn{6}{|c|}{ Total population $(n=174)$} \\
\hline & \multicolumn{3}{|c|}{ UNIVARIATE } & \multicolumn{3}{|c|}{ MULTIVARIATE } \\
\hline & $\overline{\mathrm{OR}}$ & $95 \% \mathrm{Cl}$ & $P$-value & OR & $95 \% \mathrm{Cl}$ & $P$-value \\
\hline \multicolumn{7}{|l|}{ Age onset $(<12)$} \\
\hline$\geq 12 ;<40$ & 2.93 & $1.05-8.13$ & 0.0009 & 13.6 & $1.6-116.8$ & 0.0047 \\
\hline$\geq 40$ & 6.12 & $2.26-16.60$ & & 31.6 & $3.6-276.9$ & \\
\hline \multicolumn{7}{|l|}{$\mathrm{ACQ}(<1.6)$} \\
\hline$\geq 1.6 ;<2.9$ & 0.36 & $0.12-1.11$ & 0.0926 & 0.29 & $0.08-1.00$ & 0.0458 \\
\hline$\geq 2.9 ;<3.9$ & 0.46 & $0.15-1.39$ & & 0.58 & $0.16-2.08$ & \\
\hline$\geq 3.9$ & 0.23 & $0.07-0.76$ & & 0.17 & $0.05-0.63$ & \\
\hline Atopy & 0.66 & $0.35-1.20$ & 0.176 & & & NS \\
\hline \multicolumn{7}{|l|}{ Smoking (Never) } \\
\hline Current & 0.53 & $0.12-2.35$ & 0.0608 & & & NS \\
\hline Ex & 0.46 & $0.24-0.88$ & & & & \\
\hline \multicolumn{7}{|l|}{ Age $(<48)$} \\
\hline$\geq 48 ;<56$ & 1.58 & $0.66-3.75$ & 0.1448 & & & NS \\
\hline$\geq 56 ;<64$ & 0.80 & $0.34-1.87$ & & & & \\
\hline$\geq 64$ & 1.97 & $0.85-4.59$ & & & & \\
\hline $\mathrm{FEV}_{1} \%$ & 1.02 & $1.00-1.03$ & 0.029 & & & NS \\
\hline \multicolumn{7}{|l|}{ Exhaled NO (ppb) $(<25)$} \\
\hline$\geq 25 ;<50$ & 1.83 & $0.76-4.45$ & 0.0073 & & & NS \\
\hline$\geq 50$ & 4.13 & $1.70-10.00$ & & & & \\
\hline \multicolumn{7}{|c|}{ Sputum Eosinophils \% $(<0.4)$} \\
\hline$\geq 0.4 ;<3.0$ & 1.29 & $0.07-24.39$ & 0.0437 & & & NS \\
\hline$\geq 3.0 ;<21.0$ & 0.82 & $0.04-15.00$ & & & & \\
\hline$\geq 21.0$ & 15 & $1.21-185.20$ & & & & \\
\hline Sputum Neutrophils \% & 0.94 & $0.90-0.98$ & 0.003 & & & NS \\
\hline Nasal polyposis & 1.84 & $0.96-3.53$ & 0.066 & & & NS \\
\hline
\end{tabular}

OR odds ratio, $C l$ confidence interval, ACQ Asthma Control Questionnaire, $F E V{ }_{1}$ forced expiratory volume in $1 \mathrm{~s}, N O$ nitric oxide, $p p b$ parts per billion. NS not significant

asthmatics. Poor response of eosinophils to OCS in this subpopulation needs to be further investigated.

Although OCS are known to suppress eosinophils, BEC were not different between SA patients using maintenance OCS and the ones that do not. We also noticed a high prevalence of patients with high BEC $\left(>400 / \mathrm{mm}^{3}\right)$ in the OCS maintenance treated SA patients. In these patients with high BEC despite OCS maintenance, we may now offer anti-IL5 therapy to decrease the risk of exacerbation [29-31]. Persistently high peripheral blood eosinophils have been previously reported in OCS treated asthmatics [22, 32-34]. MENSA, DREAM and SIRIUS studies show high BEC (MENSA: $320 \pm 938$ to $280 \pm 987$ ); DREAM: $280 \pm 1010$ to $230 \pm 1200$; SIRIUS: $250 \pm 1245$ to $230 \pm$ $1001)$ despite variable prevalence $(25,30$ and $100 \%$, respectively) of patients on OCS maintenance therapy. Whether poor compliance or adherence [35] to treatment or corticosteroid insensitivity are the reasons for high BEC despite OCS maintenance therapy remains unclear.

The lack of measure of compliance/ adherence to treatment represents the first limitation of our study. Poor adherence to treatment is seen in about $32-56 \%$ of severe asthmatics [1]. Although compliance was not objectively measured in these severe asthmatic patients, the BSAR investigators only enrolled their patients in the registry if they considered compliance to have been satisfactory in the previous 12 months. We also cannot be absolutely sure that the reported BEC have been measured while patients were actually taking OCS. Indeed, some investigators of BSAR could have provided historical BEC (before starting chronic OCS) or could have stopped OCS in order to obtain a blood eosinophils level > $300 / \mathrm{mm} 3$, required for the reimbursement of anti-IL5 in Belgium. Nevertheless, at least the major contributing 
centers have confirmed that patients were already on OCS maintenance therapy at the time of blood sample collection. Another limitation of our study is the fairly low number of sputums due to the fact that Liège is the only center to use sputum in routine checkup. This could be skewing the data in favor of neutrophilic phenotype for low BEC group, although it is well established that low BEC can have high sputum eosinophils.

In our study, in a small population of patients with eosinophilia despite OCS treatment, we found normalization of BEC under anti-IL5 therapy. With antiIL5 therapy, a reduction in blood eosinophils is achieved, even in those patients who presented persistent eosinophilia under OCS therapy [36]. More importantly, the introduction of an anti-IL5 treatment allowed an opportunity to reduce the OCS dose in these patients.

Surprisingly, in a fairly small number of patients of this cohort of severe asthmatics, highly uncontrolled asthma was not associated with a high BEC as we could have expected. Instead, it was characterized by impaired lung function (low $\mathrm{FEV}_{1}$ ) and diffusion capacity, and higher rate of emphysema (52\%). Emphysema which is associated with lower diffusion capacity, may be a cause of dyspnea, which is a symptom considered in the evaluation of asthma control. These subjects fulfill the profile of patients with Asthma COPD overlap. In contrast, as previously reported [25], some patients with a late-onset eosinophilic phenotype present with low symptom expression despite high inflammation. These are poor perceivers with overestimated asthma control, but are at risk for exacerbations.

\section{Conclusion}

Approximately one-fifth of SA patients were treated with maintenance OCS at enrollment in BSAR. We demonstrated a significant association between late-onset, exacerbations, non-atopic status and chronic use of OCS in this population. Almost half of SA patients treated with chronic OCS have BEC $\geq 400 / \mathrm{mm}^{3}$. Moreover, we show a significant association between high $\mathrm{BEC}$ and lateonset asthma in OCS maintenance SA patients.

\section{Supplementary information}

Supplementary information accompanies this paper at https://doi.org/10. 1186/s12931-020-01460-7.

Additional file 1: Table S1. Comparison of SA patients on biologics (anti-IL5 or anti-lgE) with or without chronic OCS treatment $(n=270)$.

\section{Abbreviations}

ACQ: Asthma control questionnaire; ATS: American Thoracic Society; BEC: Blood Eosinophil count; BSAR: Belgian Severe Asthma registry; EGPA: Eosinophilic granulomatosis with polyangiitis, formerly called ChurgStrauss syndrome; ERS: European Respiratory Society; FENO: Fractional exhaled nitric oxide; FEV 1 : Forced expiratory volume in $1 \mathrm{~s}$; FVC: Functional vital capacity; GERD: Gastroesophageal reflux; IL: Interleukin; OCS: Oral corticosteroids; SA: Severe asthma
Acknowledgements

Not applicable.

\section{Authors' contributions}

Graff S and Schleich F had full access to all of the data in the study and take responsibility for the integrity of the data and the accuracy of the data analysis. Graff S, Schleich F, Vanwynsberghe S, Brusselle G, Hanon S, Sohy C, Dupont L, Peche R, Michils A, Pilette C, Joos G, and Louis R contributed substantially to the study design, data analysis and interpretation, and the writing of the manuscript. The author(s) read and approved the final manuscript.

\section{Funding}

This work was supported by the European Union (Interreg EMR Meuse Rhine 5a) and Interuniversity Attraction Poles Program (IUAP), Belgian Science Policy $(\mathrm{P} 7 / 30)$

\section{Availability of data and materials}

The datasets used and/or analyzed during the current study are available from the corresponding author on reasonable request.

Ethics approval and consent to participate CHU Liège: 2008/221.

Consent for publication

Not applicable.

\section{Competing interests}

Dr. Graff has nothing to disclose.

Dr. Vanwynsberghe has nothing to disclose.

Dr. Brusselle reports personal fees from Astra Zeneca, personal fees from Boehringer-Ingelheim, personal fees from Chiesi, personal fees from GlaxoSmithKline, personal fees from Novartis, personal fees from Sanofi, personal fees from Teva, outside the submitted work.

Dr. Hanon has nothing to disclose.

Dr. Sohy has nothing to disclose.

Dr. Dupont has nothing to disclose.

Dr. Peche has nothing to disclose.

Dr. Michils reports grants, personal fees and other from Astrazeneca, grants, personal fees and other from Chiesi, grants and personal fees from GSK, personal fees from Novartis, outside the submitted work.

Dr. Pilette has nothing to disclose.

Dr. JOOS reports grants from AstraZeneca, personal fees from Bayer, grants from Chiesi, personal fees from Eureca vzw, grants and personal fees from GlaxoSmithKline, personal fees from Teva, outside the submitted work; all fees were paid to the department.

Dr. LOUIS reports grants and personal fees from GSK, grants and personal fees from AZ, grants and personal fees from Novartis, grants from Chiesi, outside the submitted work.

Dr. Schleich has nothing to disclose.

\section{Author details}

1Department of Respiratory Medicine, CHU Sart-Tilman, I3GIGA Research Group, University of Liege, Liege, Belgium. '2Department of Respiratory Medicine, Ghent University Hospital, Ghent, Belgium. ${ }^{3}$ Respiratory Division, Universitair Ziekenhuis Brussel, Vrije Universiteit Brussel (VUB), Brussels, Belgium. ${ }^{4}$ Department of Respiratory Medicine, Centre Hospitalier Universitaire UCL Namur, Université Catholique de Louvain, Yvoir, Belgium. ${ }^{5}$ Department of Respiratory Medicine, Katholieke Universiteit Leuven, Leuven, Belgium. ${ }^{6}$ Department of Respiratory Medicine, CHU-Charleroi, A. Vésale Hospital, Charleroi, Belgium. ${ }^{7}$ Chest Department, Erasme University Hospital, Université Libre de Bruxelles, Brussels, Belgium. ${ }^{8}$ Cliniques Universitaires St-Luc and Institute of Experimental and Clinical Research, Université Catholique de Louvain, Brussels, Belgium. 
Received: 28 May 2020 Accepted: 17 July 2020

Published online: 12 August 2020

\section{References}

1. Chung KF, Wenzel SE, Brozek JL, Bush A, Castro M, Sterk PJ, et al. International ERS/ATS guidelines on definition, evaluation and treatment of severe asthma. Eur Respir J. 2014;43:343-73.

2. Accordini S, Corsico A, Cerveri I, Gislason D, Gulsvik A, Janson C, et al. The socio-economic burden of asthma is substantial in Europe: the burden of asthma in Europe. Allergy. 2007;63:116-24.

3. Chen W, Safari A, FitzGerald JM, Sin DD, Tavakoli H, Sadatsafavi M. Economic burden of multimorbidity in patients with severe asthma: a 20-year population-based study. Thorax. 2019;74:1113-9.

4. van Bragt JJMH, Adcock IM, Bel EHD, Braunstahl G-J, ten Brinke A, Busby J, et al. Characteristics and treatment regimens across ERS SHARP severe asthma registries. Eur Respir J. 2020;55:1901163.

5. Casan Clarà P, Martínez González C. Accumulated Dose of Systemic Corticosteroids: Significant Medical Information. Arch Bronconeumol. 2020

6. Chen W, Lynd LD, FitzGerald JM, Marra CA, Balshaw R, To T, et al. Excess medical costs in patients with asthma and the role of comorbidity. Eur Respir J. 2016;48:1584-92.

7. Ramsahai JM, Wark PA. Appropriate use of oral corticosteroids for severe asthma. Med J Aust. 2018;209:18-21.

8. Barnes PJ, Adcock IM. How do corticosteroids work in asthma? Ann Intern Med. 2003;139:359.

9. James A, Gyllfors $\mathrm{P}$, Henriksson E, Dahlén SE, Adner M, Nilsson G, et al. Corticosteroid treatment selectively decreases mast cells in the smooth muscle and epithelium of asthmatic bronchi. Allergy. 2012;67:958-61.

10. Schleich F, Brusselle G, Louis R, Vandenplas O, Michils A, Pilette C, et al. Heterogeneity of phenotypes in severe asthmatics. The Belgian severe asthma registry (BSAR). Respir Med. 2014;108:1723-32.

11. Fahy J. Proceedings of the ATS workshop on refractory asthma. Am J Respir Crit Care Med. 2000;162:2341-51.

12. GINA-Global Initiative for Asthma. Global Strategy for Asthma Management and Prevention, 2018. Available from: www.ginasthma.org.

13. Louis R, Sele J, Henket M, Cataldo D, Bettiol J, Seiden L, et al. Sputum eosinophil count in a large population of patients with mild to moderate steroid-naive asthma: distribution and relationship with methacholine bronchial hyperresponsiveness. Allergy. 2002;57:907-12.

14. Juniper EF, Guyatt GH, Epstein RS, Ferrie PJ, Jaeschke R, Hiller TK. Evaluation of impairment of health related quality of life in asthma: development of a questionnaire for use in clinical trials. Thorax. 1992;47:76-83.

15. Juniper EF, O'Byrne PM, Guyatt GH, Ferrie PJ, King DR. Development and validation of a questionnaire to measure asthma control. Eur Respir J. 1999; 14:902-7.

16. Schatz M, Sorkness CA, Li JT, Marcus P, Murray JJ, Nathan RA, et al. Asthma control test: reliability, validity, and responsiveness in patients not previously followed by asthma specialists. J Allergy Clin Immunol. 2006:117:549-56.

17. Dweik RA, Boggs PB, Erzurum SC, Irvin CG, Leigh MW, Olin A-C, et al. An official ATS clinical practice guideline: interpretation of exhaled nitric oxide levels (FENO) for clinical applications. Am J Respir Crit Care Med. 2011;184:602-15.

18. Guiot J, Demarche S, Henket M, Paulus V, Graff S, Schleich F, et al. Methodology for Sputum Induction and Laboratory Processing. J Vis Exp. 2017; [cited 2019 Dec 9]; Available from: https://www.jove.com/ video/56612/methodology-for-sputum-induction-and-laboratory-processing.

19. Louis R, Godinas L, Schleich F. Induced sputum - towards normal values, non invasive assessment of airways inflammation in asthma and COPD. 14th, tetrapoleos street, Athens, 115 27. Greece: Paschalidis Medical Publications; 2011

20. Bjelland I, Dahl AA, Haug T, Neckelmann D. The validity of the hospital anxiety and depression scale: an update d literature review. J Psychosom Res. 2002;52:69-77.

21. Nair P, Wenzel S, Rabe KF, Bourdin A, Lugogo NL, Kuna P, et al. Oral glucocorticoid-sparing effect of Benralizumab in severe asthma. N Engl J Med. 2017:376:2448-58.

22. Bel EH, Wenzel SE, Thompson PJ, Prazma CM, Keene ON, Yancey SW, et al. Oral glucocorticoid-sparing effect of mepolizumab in eosinophilic asthma. $\mathrm{N}$ Engl J Med. 2014;371:1189-97.

23. Cataldo D, Louis R, Michils A, Peché R, Pilette C, Schleich F, et al. Severe asthma: oral corticosteroid alternatives and the need for optimal referral pathways. J Asthma Off J Assoc Care Asthma. 2020:1-11.
24. Schleich FN, Manise M, Sele J, Henket M, Seidel L, Louis R. Distribution of sputum cellular phenotype in a large asthma cohort: predicting factors for eosinophilic vs neutrophilic inflammation. BMC Pulm Med. 2013;13:11.

25. Haldar P, Pavord ID, Shaw DE, Berry MA, Thomas M, Brightling CE, et al. Cluster analysis and clinical asthma phenotypes. Am J Respir Crit Care Med. 2008;178:218-24.

26. Green RH, Brightling CE, McKenna S, Hargadon B, Parker D, Bradding P, et al. Asthma exacerbations and sputum eosinophil counts: a randomised controlled trial. Lancet. 2002;360:1715-21.

27. Berthon BS, Gibson PG, Wood LG, MacDonald-Wicks LK, Baines KJ. A sputum gene expression signature predicts oral corticosteroid response in asthma. Eur Respir J. 2017:49:170-80.

28. Demarche S, Schleich F, Henket M, Paulus V, Louis R, Van Hees T. Step-down of inhaled corticosteroids in non-eosinophilic asthma: a prospective trial in real life. Clin Exp Allergy J Br Soc Allergy Clin Immunol. 2018;48:525-35.

29. Schleich FN, Chevremont A, Paulus V, Henket M, Manise M, Seidel L, et al. Importance of concomitant local and systemic eosinophilia in uncontrolled asthma. Eur Respir J. 2014;44:97-108.

30. Price D, Wilson AM, Chisholm A, Rigazio A, Burden A, Thomas M, et al. Predicting frequent asthma exacerbations using blood eosinophil count and other patient data routinely available in clinical practice. J Asthma Allergy. 2016;9:1-12.

31. Kerkhof M, Tran TN, van den Berge M, Brusselle GG, Gopalan G, Jones RCM, et al. Association between blood eosinophil count and risk of readmission for patients with asthma: historical cohort study. PLoS One. 2018;13: e0201143.

32. Ortega HG, Liu MC, Pavord ID, Brusselle GG, FitzGerald JM, Chetta A, et al. Mepolizumab treatment in patients with severe Eosinophilic asthma. N Engl Med. 2014;371:1198-207.

33. Pavord ID, Korn S, Howarth P, Bleecker ER, Buhl R, Keene ON, et al. Mepolizumab for severe eosinophilic asthma (DREAM): a multicentre, double-blind, placebo-controlled trial. Lancet Lond Engl. 2012;380:651-9.

34. Bjerrum AS, Skjold T, Schmidt J. Baseline characteristics of 36 mepolizumabpatients. Experiences from the clinical practice. Eur Respir J. 2017:50:PA3588.

35. Hassan M, Davies SE, Trethewey SP, Mansur AH. Prevalence and predictors of adherence to controller therapy in adult patients with severe/difficult-totreat asthma: a systematic review and meta-analysis. J Asthma Off J Assoc Care Asthma. 2019:1-10.

36. Farne HA, Wilson A, Powell C, Bax L, Milan SJ. Anti-IL5 therapies for asthma. Cochrane Database Syst Rev. 2017;9:CD010834.

\section{Publisher's Note}

Springer Nature remains neutral with regard to jurisdictional claims in published maps and institutional affiliations.

Ready to submit your research? Choose BMC and benefit from:

- fast, convenient online submission

- thorough peer review by experienced researchers in your field

- rapid publication on acceptance

- support for research data, including large and complex data types

- gold Open Access which fosters wider collaboration and increased citations

- maximum visibility for your research: over $100 \mathrm{M}$ website views per year

At BMC, research is always in progress.

Learn more biomedcentral.com/submissions 